\title{
BIOPSIA RENAL POR ABORDAJE RETROPERITONEOSCÓPICO: NUESTRA EXPERIENCIA EN 53 PACIENTES PEDIÁTRICOS.
}

\author{
Rafael Luque Mialdea 1,2, Rosa Martín-Crespo Izquierdo', Luis Díazl, Angustias Fernández³, \\ Dolores Morales ${ }^{3}$ y Jesús Cebrían 4 . \\ 'Servicio de Cirugía Pediátrica Complejo Hospitalario de Toledo \\ 2Sección de Urología Pediátrica. Servicio Cirugía Pediátrica Hospital General Universitario Gregorio Marañón. \\ ${ }^{3}$ Nefrología. Departamento Pediatría. Hospital General Universitario Gregorio Marañón. \\ ${ }^{4}$ Sección de Anestesiología Pediátrica. Hospital General Universitario Gregorio Marañón. Madrid. España.
}

\begin{abstract}
Resumen.- OBJETIVO: El abordaje retroperitoneoscópico posibilita una adecuada visualización de la fosa renal y de las estructuras retroperitoneales. Planteamos como objetivo la realización de biopsia renal por retroperitoneoscopia en aquellos Servicios de Cirugía Pediátrica en los que se realiza por cirugía convencional.

MÉTODOS: Hemos realizado 53 biopsias renales por abordaje retroperitoneoscopico Se realizaron en $28 \mathrm{va}$ rones y 25 mujeres, de edades entre los 13 meses y 19 años ( rango medio de edad de 4 años). Los diagnósticos por los que sé biopsió fueron: Síndrome nefrótico $(n=20)$; Síndrome hemolítico-urémico( $n=3)$; Hematuria $(n=15)$; Purpura idiopàtica (2) i proteinuría (n=13), de estos pacientes, 10 estaban en insuficiencia renal. En todos los casos el abordaje retroperitoneoscópico se realizó según nuestra técnica.
\end{abstract}

RESULTADOS: En todos los casos la exposición del riñón fue adecuada y la toma de biopsia concluyente con un diagnóstico anatomopatológico, realizándose pruebas de immunohistoquímica y estudio morfológico de la muestra. Se reconvirtió 1 caso por sangrado del lecho renal. En 51 casos el sangrado del procedimiento no alcanzó los 20 cc. No se precisó drenaje postoperatorio. La estancia media fue en los primeras 20 casos de 48 horas y en el resto de 24 a 36 horas.

CONCLUSIONES: La biopsia renal retroperitoneoscópica es adecuada para la edad pediátrica siendo una alternativa real de la cirugía abierta y en las situaciones de contraindicación de la biopsia percutánea ecodirigida por: 1. Excelente exposición del riñón. 2. Adecuada cantidad de tejido renal para estudio. 3. Mínima morbilidad intra y postoperatoria. 4. Disminución de estancias hospitalarias con incorporación precoz a la vida cotidiana.

Palabras clave: Biopsia renal. Retroperitoneoscopia. Biopsia renal por abordaje retroperitoneoscopico. Edad pediátrica.

Summary.- OBJECTIVES: The retroperitoneoscopic approach enables adequate vision of the renal fossa and retroperitoneal structures. Our objective is to support the performance of renal biopsies through a retroperitoneoscopic approach in those pediatric surgery departments still doing open biopsies.

METHODS: We performed 53 renal biopsies through a retroperitoneoscopic approach. Twenty-eight patients were males and 25 females, with ages between 13 months and 19 years (mean age range 4 years). The biopsies were indicated after the following diagnosis: 
nephrotic syndrome ( $n=20)$, hemolytic-uremic syndrome $(n=3)$, hematuria $(n=15)$, idiopathic purpura $(n=2)$, proteinuria $(n=13)$. Ten patients were in renal failure. We performed our technique of retroperitoneoscopic approach in all cases.

RESULTS: Adequate exposure of the kidney was achieved in all cases, and the biopsy sample gave a pathologic diagnosis after immunohistochemical and morphologic studies. One case was converted to open surgery due to bleeding from the kidney. In 51 cases the estimated blood loss was lower than 20 cc. No drainage was necessary after surgery. Mean hospital stay was 48 hours for the first 20 cases and between 24-36 hours for the remainder.

CONCLUSIONS: The Retroperitoneoscopic renal biopsy is an adequate procedure for the pediatric patients and represents a real alternative to open biopsy, and in cases of contraindication for ultrasound guided percutaneous biopsy, because: 1. It offers an excellent exposure of the kidney. 2. It obtains a kidney sample enough for the study. 3. The morbidity associated with the procedure is minimal. 4. Diminishes the hospital stay and shortens the time to return back to normal life.

Keywords: Renal biopsy. Retroperitoneoscopy. Retroperitoneoscopic approach renal biopsy. Children.

\section{INTRODUCCIÓN}

La biopsia renal es un procedimiento necesario para el diagnóstico y pronóstico de patologías renales, con o sin insuficiencia renal. Desde la década de los 80, el método de elección es la biopsia renal percutánea ecodirigida en la edad pediátrica (1-7). Pero han descrito contraindicaciones, relativas y absolutas, para la biopsia renal percutánea ecodirigida (8-12), que necesita la realización de la biopsia por cirugía convencional: coagulopatías, discrasia sanguínea, riñón solitario (con la excepción de injerto renal), hipertensión arterial severa mal controlada, aneurisma de la arteria renal, riñón alto, obesidad, mala localización renal por ecografía, fallo de la punción percutánea ecodirigida y falta de colaboración del paciente. La cirugía convencional se realiza por incisión de lumbotomía clásica o modificada por minilumbotomía (13), dorsal o lateral, con la desventaja de que es un procedimiento invasivo con estancia prolongada en el hospital y un índice de complicaciones, locales y generales (14), que hacen poco atractivo su uso, como método diagnóstico. Exponemos nuestra experiencia en la realización de la biopsia renal mediante abordaje retroperitoneoscópico utilizando la técnica del balón de distensión para la disección retroperitoneal $(8,15,16)$ como alternativa tanto para aquellos servicios que la realizan sistemáticcamente por cirugía convencional como en aquellos casos en los que la biopsia renal percutánea ecodirigida no esté indicada.

\section{MATERIAL Y MÉTODOS}

Desde el año de 1995 hemos realizado 53 biopsias renales por abordaje retroperitoneoscopico mediante técnica del balón de disección. Varones 28 y mujeres 25, con edad media de 4 años (rango de edad 13 meses y 19 años). En 10 pacientes con insuficiencia renal en grado variable. Los diagnósticos por los que se indicó realizar biopsia renal fueron: Síndrome nefrótico ( $n=20)$; Síndrome hemolítico-urémico $(n=3)$; Hematuria $(n=15)$, Purpura idiopàtica $(n=2)$ y proteinuría $(n=13)$.

El abordaje retroperitoneoscópico fue realizado con el paciente en posición de lumbotomía situado hacia posterior y bajo anestesia general. No drenaje vesical ni gástrico. El inicio del abordaje fue abierto con utilización de 3 trocares en 47 casos y 2 trocares en 6 casos.

La técnica habitual de nuestro abordaje retorperitoneoscópico consistió en incisión de $1 \mathrm{~cm}$. a nivel del ángulo conformado por la $12^{\circ}$ costilla y la masa lumbar, y posterior disección digital hasta la fascia de la Gerota, apertura de la misma y una vez palpado el polo renal inferior, se introdujo el balón de disección procediéndose a disecar el retroperitoneo mediante insuflación de 150 a $200 \mathrm{cc}$ de aire, hasta obtener un adecuado espacio retroperitoneal.

Tras la retirada del balón de insuflación e introducción de trocar de Hasson se realizó retroneumoperitoneo, con presión media de $11 \mathrm{~mm}$ de $\mathrm{Hg}$. Bajo visión directa se introdujeron el resto de trocares, por incisión de $5 \mathrm{~mm}$, en disposición triangular con respecto al primer trocar. La toma de biopsia se obtuvo del polo renal inferior, generalmente del riñón derecho, mediante tijera realizando el corte y la electrocoagulación de la periferia de la corteza renal en el mismo acto y posteriormente electrocoagulación bipolar hemostática, tanto periférica como del lecho renal.

Tras hemostasia del lecho se retiran los trocares y cierran los puertos. En todos los casos se infiltró con bupivacaina $0.25 \%$ los puertos y se cerraron las heridas incisionales con aplicación de sutura o bien pegamento. No se dejaron drenajes. 


\section{RESULTADOS}

En los 53 casos la exposición del riñón fue adecuada y la toma de biopsia concluyente con un diagnóstico anatomopatológico en el $100 \%$ de los casos, realizándose estudio histológico, immunohistoquímica y estudio por microscopia electrónica. En ningún caso la electrocoagulación de la periferia de la muestra de la biopsia impidió o alteró el resultado diagnóstico. El tiempo medio quirúrgico fue de 45 minutos (30-90 $\mathrm{min}$.). En toda nuestra serie tuvimos complicación de sangrado en 2 pacientes.

En 1 paciente precisó conversión (1.3\%), su causa fue por sangrado del lecho renal, aunque la toma ya se había extraído, en este caso fue necesario realizar una minilumbotomía, por ampliación de unos de los puertos de entrada de $1 \mathrm{~cm}$.

En el otro caso, una vez finalizada la cirugía presenta hematoma de un puerto incisional que precisó revisión en quirófano.
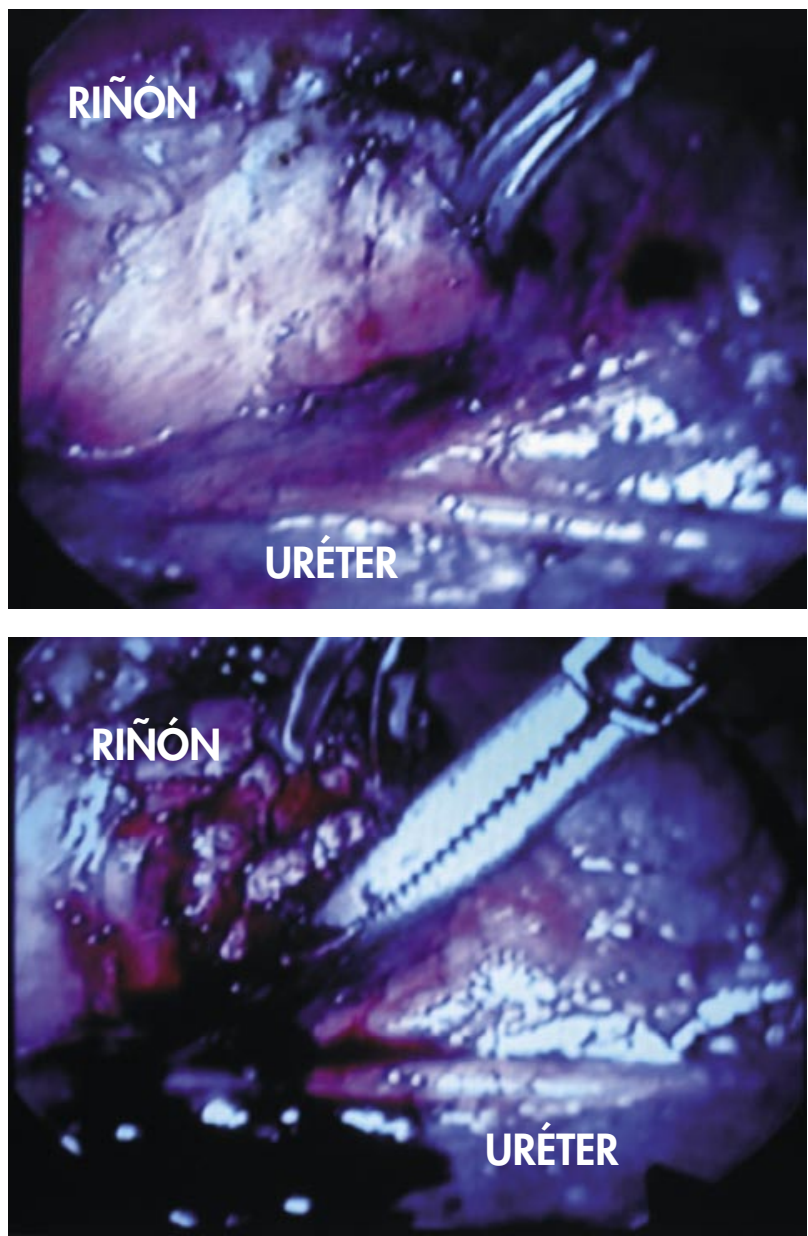

En el resto de los 51 casos, el sangrado del procedimiento no alcanzó los 20 cc. No se precisó drenaje postoperatorio, destacando la ausencia de sangrado del lecho renal. En nuestra serie no hubo complicaciones en el postoperatorio inmediato (locales - infección de puertos, hematomas, hernia- ni generales - hematuria macroscópica, complicaciones respiratorias-), ni tardío. No hubo descenso del hematocrito relevante en toda nuestra serie, y ningún paciente requirió transfusión sanguínea. La estancia media fue en los primeras 20 casos de 48 horas y en el resto ha sido de 24 a 36 horas.

Destacamos la ausencia de complicaciones postoperatorias generales, siendo los controles ecográficos posteriores normales.

\section{DISCUSIÓN}

Los estudios histopatológicos mantienen su estándar para el diagnóstico de enfermedad renal
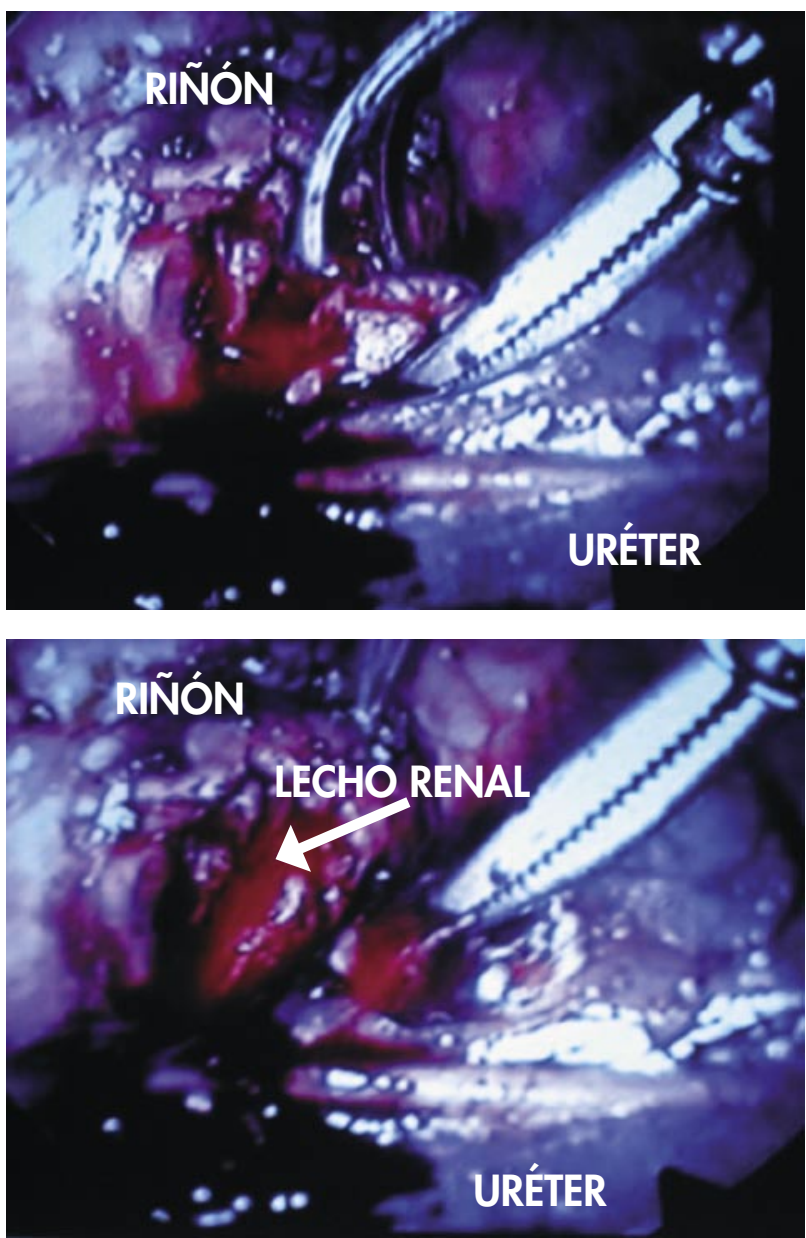

FIGURA 1. Imágenes de la biopsia renal por retroperitoneoscopia. 
con o sin insuficiencia renal. La biopsia renal ecodirigida se establece como método de elección, aún en la edad pediátrica, con analgesia local o sedación-analgesia, desde la década de los 80 (1-7). La existencia de contraindicación absoluta o relativa del procedimiento en pacientes de alto riesgo (8-12), precisa de la cirugía convencional como método diagnóstico alternativo.

En nuestra experiencia, que hasta 1995 la biopsia renal la realizábamos sistemáticamente por cirugía convencional, el abordaje retroperitoneoscópico $(8,15)$ nos ofreció una clara alternativa, fundamentalmente en aquellos pacientes de alto riesgo para la biopsia renal percutánea ecodirigida.

En todos nuestros pacientes, la cantidad de tejido renal obtenida mediante disección con corte y electrocoagulación de tijera, fue optimo para el $100 \%$ de diagnósticos histopatológicos, sin que la electrocoagulación alterara el resultado de la misma. La realización del abordaje retroperitoneoscopio para la realización de nefrectomía (17), nos ofreció un método alternativo de la cirugía abierta para la biopsia renal, nuestra metodología a diferencia de otros autores la realizamos siempre mediante tijera y tres trocares, habiéndose descrito la realización de la misma con tres $(8,15)$, dos $(8,10-12)$ y un único trocar (18), con pinzas de fórceps para biopsia.

En nuestra serie, tuvimos una morbilidad intraoperatoria del $1.3 \%$ por sangrado, que conllevó a su conversión y un $1.3 \%$ de hematoma postcirugía que se debió a sangrado de la pared del $2^{\circ}$ puerto y necesitó reexploración quirúrgica. En ambos casos no hubo repercusión en el hematocrito ni en la estancia hospitalaria postoperatoria.

Destacamos que no hemos tenido complicaciones postoperatorias secundarias al procedimiento biopsico, a diferencia de las descritas tanto en cirugía convencional (8) como en realizados por punción percutánea ecodirigida (19-23): hematoma renal o perirenal, hematuria macroscópica, infección de herida, íleo intestinal, neumotorax y atelectasia, nefrectomía, fístula arteriovenosa renal. El abordaje retroperitoneoscópico ofrece todas las ventajas inherentes de éste tipo de procedimiento con respecto a la cirugía abierta (Tabla I), y además nos ha ofrecido una visión renal general del retroperitoneo con visión directa de la zona de la biopsia, así como las características macroscópicas renales y en nuestra experiencia, como en la de otros autores $(8-12,16,18)$, ofrece la misma eficacia para la obtención de tejido renal que la cirugía abierta, convencional o modificada, pero siendo menos invasivo el procedimiento.

Nuestra conclusión es que desde que el abordaje retroperitoneoscópico fue utilizado para la realización de biopsia renal en la edad pediátrica (8), podemos decir que es un método de abordaje adecuado para sustituir la cirugía abierta en pacientes en los que la biopsia renal percutánea ecodirigida esté contraindicada, o bien en aquellos servicios en los que se realiza de forma sistemática por cirugía abierta. El abordaje retroperitoneoscópico consigue una excelente exposición del riñón, adecuada cantidad de tejido renal para estudio anatomopatológico y una baja tasa de morbilidad por complicaciones intra o postoperatorias, ausencia de pérdida de ri-

\section{TABLA I. VENTAJAS DE RETROPERITONEOSCOPIA EN COMPARACIÓN CON CIRUGÍA CONVENCIONAL.}

- Manejo quirúrgico igual e incluso mejor que cirugía convencional

- Respeto por pared muscular

\begin{tabular}{l|l} 
- Incisiones pequeñas & Menor dolor postoperatorio \\
\cline { 2 - 2 } & Movilización precoz y reducción de complicaciones respiratorias \\
\hline
\end{tabular}

- Descenso complicaciones locales herida quirúrgica con menor número de reintervenciones.

- Reducción de estancias hospitalarias

- Reincorporación a la actividad normal precoz- escuela, hogar-

- Reducción de costes por procesos quirúrgicos 
ñón secundaria al procedimiento y disminución con respecto a la cirugía convencional de estancia hospitalaria, siendo similar a la estancia en los casos de biopsia percutánea ecodirigida.

\section{BIBLIOGRAFIA y LECTURAS RECOMENDADAS (*lectura de interés $y^{* *}$ lectura fundamental)}

*1. CHAN, J.C.; BREWER, W.H.; STILL, W.J.: "Renal biopsies under ultrasound guidance: 100 consecutive biopsies in children's Ural". 129: 103, 1983.

2. BACHMANN, H.; HECKEMANN, R.; OLBING, H.: "Percutaneous renal biopsy in children under guidance of ultrasonic real time technique". Int. J. Pediatr. Nephrol., 5: 175, 1984.

*3. NADEL, L.; BAUMGARTNER, B.R.; BERNARDINO, M.E.: "Percutaneous renal biopsies: accuracy, safety, and indications". Urol. Radiol., 8: 67, 1986.

4. FENEBERG, R.; SCHAEFER, F.; ZIEGER, B. y cols.: "Percutaneous renal biopsy in children: a 27-year experience". Nephron, 79: 438, 1998.

5. SAHNEY, S.; MOHAN, G.C.: "Renal biopsy in infants and children". Am. J. Kidney Dis., 23: 31, 1994.

6. WEBB, N.J.; PEREIRA, J.K.; CHAIT, P.G. y cols.: "Renal biopsy in children: comparison of two techniques". Pediatr. Nephrol., 8: 486, 1994.

**7. BOHLIN, A.B.; EDSTROM, S.; ALMGREN, B. y cols.: "Renal biopsy in children: indications, technique and efficacy in 119 consecutive cases". Pediatr. Nephrol., 9: 201, 1995.

**8. GAUR, D.R.; AGARWAL, M.V.; KHOCHIKAR, V.y cols.: "Laparoscopic renal biopsy via retroperitoneal approach". 151: 925, 1994.

*9. SHETYE, K.R.; KAVOUSSI, L.R.; RAMAKUMAR, S. y cols.: "Laparoscopic renal biopsy: a 9year experience”. BJU International, 817, 2003.

*10. CAIONE, P.; MICALI, S.; RINALDI, S. y cols.: "Retroperitoneal laparoscopy for renal biopsy in children”. J. Urol., 164: 1080, 2000.
*11. GIMENEZ, L.F.; MICALI, S.; CHEN, R.N. y cols.: "Laparoscopic renal biopsy". Kidney Int., 54: 525, 1998.

12. MUKHTAR, Z.; STEINBRECHER, H.; GILBERT, R.D.; y cols.: "Laparoscopic renal biopsy in obese children”. Pediatr. Nephrol., 20: 495, 2005.

*13. THOMAS, J.H.; PIERCE, G.E.; HERMRECK, A.S.: "Open renal biopsy. Surgical technique and results”. Am. Surg., 49: 400, 1983.

14. ALMKUIST, R.; BUKALEW, V.M. Jr.: "Techniques of renal biopsy". Urol. Clin. N. Amer., 6: 503, 1979.

15. LUQUE MIALDEA, R.; MARTIN-CRESPO, R.; BARRIENTOS, G. y cols.: "Indications from the laparoscopic approach in pediatric urological surgery: our experience in 49 patients". Actas Urol. Esp., 23: 483, 1999.

**16. LUQUE MIALDEA, R.; MARTIN-CRESPO, R.: "Laparoscopia en Urología Pediátrica". Arch. Esp. Urol., 55: 737, 2002.

17. LUQUE MIALDEA, R.; MARTIN-CRESPO, R.; NAVASCUES DEL RIO, J.A. y cols.: "Nefrectomia laproscópica retroperitoneal en la infancia". Act. Urol. Esp., 21: 637, 1997.

18. MARTINO, A.; ZAMPARELLI, M.; COBELLIS, G. y cols.: "One-trocar surgery: a less invasive videosurgical approach in childhood". J. Pediatr. Surg., 36: 811, 2001.

**19. DE MEDINA MORALES, F.; VIDAL CASTRO, M.A.; MOTA HERNANDEZ, F.: "Technic and complications of percutaneous renal biopsy". Bol. Med. Hosp. Infant. Mex., 35: 397, 1978.

20. PFISTER, R.C.; NEWHOUSE, J.H.; YODER, I.C. y cols.: "Complications of pediatric percutaneous renal procedures: incidence and observations". Urol. Clin. North Am., 10: 563, 1983.

21. KAMITSUJI, H.; YOSHIOKA, K.; ITO, H.: "Percutaneous renal biopsy in children: survey of pediatric nephrologists in Japan". Pediatr. Nephrol., 13: 693, 1999.

22. BOGDANOVIC, R.; OGNJENOVIC, M.; CVORIC, A. y cols.: "Percutaneous biopsy of the kidney in children: indications, results, and complications". Srp. Arh. Celok. Lek., 118: 243, 1990.

23. GRAU, J.H.; GONICK, P.; WILSON, A.: "Postbiopsy intrarenal arteriovenous fistula". J. Urol., 122: $233,1979$. 